\title{
The atmospheric parameters of FGK stars using wavelet analysis of CORALIE spectra
}

\author{
S. Gill, P. F. L. Maxted, and B. Smalley \\ Astrophysics Group, Keele University, Keele ST5 5BG, UK \\ e-mail: s.gill@keele.ac.uk
}

Received 14 September 2017 / Accepted 18 January 2018

\begin{abstract}
Context. Atmospheric properties of F-, G- and K-type stars can be measured by spectral model fitting or with the analysis of equivalent width (EW) measurements. These methods require data with good signal-to-noise ratios (S/Ns) and reliable continuum normalisation. This is particularly challenging for the spectra we have obtained with the CORALIE échelle spectrograph for FGK stars with transiting M-dwarf companions. The spectra tend to have low S/Ns, which makes it difficult to analyse them using existing methods.

Aims. Our aim is to create a reliable automated spectral analysis routine to determine $T_{\mathrm{eff}},[\mathrm{Fe} / \mathrm{H}], V \sin i$ from the CORALIE spectra of FGK stars.

Methods. We use wavelet decomposition to distinguish between noise, continuum trends, and stellar spectral features in the CORALIE spectra. A subset of wavelet coefficients from the target spectrum are compared to those from a grid of models in a Bayesian framework to determine the posterior probability distributions of the atmospheric parameters.

Results. By testing our method using synthetic spectra we found that our method converges on the best fitting atmospheric parameters. We test the wavelet method on 20 FGK exoplanet host stars for which higher-quality data have been independently analysed using EW measurements. We find that we can determine $T_{\text {eff }}$ to a precision of $85 \mathrm{~K},[\mathrm{Fe} / \mathrm{H}]$ to a precision of 0.06 dex and $V \sin i$ to a precision of $1.35 \mathrm{~km} \mathrm{~s}^{-1}$ for stars with $V \sin i \geq 5 \mathrm{~km} \mathrm{~s}^{-1}$. We find an offset in metallicity $\approx-0.18$ dex relative to the EW fitting method. We can determine $\log g$ to a precision of 0.13 dex but find systematic trends with $T_{\text {eff }}$. Measurements of $\log g$ are only reliable enough to confirm dwarf-like surface gravity $(\log g \approx 4.5)$.

Conclusions. The wavelet method can be used to determine $T_{\mathrm{eff}}$, [Fe/H], and $V \sin i$ for FGK stars from CORALIE échelle spectra. Measurements of $\log g$ are unreliable but can confirm dwarf-like surface gravity. We find that our method is self consistent, and robust for spectra with $S / N \gtrsim 40$.
\end{abstract}

Key words. stars: atmospheres

\section{Introduction}

The WASP project (Wide Angle Search for Planets; Pollacco et al. 2006) monitors the night sky in search of exoplanet transit signatures around bright and nearby stars. So far, WASP has discovered over $130^{1}$ exoplanet systems and continues to be the origin of many exoplanet discoveries. The SuperWASP instruments are sensitive to Jovian planets transiting solar-type stars which periodically attenuates the brightness of the host. Candidate exoplanet systems are subject to radial velocity measurements from the CORALIE échelle spectrograph (Queloz et al. 2000) to determine whether the transiting companion is indeed of planetary mass. Occasionally, these measurements yield radial velocity differences of tens of $\mathrm{km} \mathrm{s}^{-1}$ over an orbit, suggesting that the transit is stellar in origin as opposed to Jovian. These are typically low-mass stars $\left(\leq 0.5 M_{\odot}\right)$ which have radii comparable to large planets, hence mimicking an exoplanet transit signal very well. These systems are given the flag "EBLM" (eclipsing binary, low mass) in the database used to coordinate observations to exclude them from the planet-hunting process.

M-dwarfs $\left(0.08 \leq \mathrm{M} \leq 0.6 M_{\odot}\right)$ are the most abundant stars in the Galaxy (Henry et al. 2006). They slowly descend to the main sequence along a near-vertical Hyashi track

1 TEPCAT (Southworth 2011). where they can remain on the main sequence for billions of years. Those which are small enough to be fully convective restrict the build-up of helium ash in their cores, resulting in main sequence lifetimes greater than the age of the Universe (Baraffe et al. 1998).

Numerous photometric measurements around the infrared region are required to sample the peak of the spectral energy distribution (SED) of M-dwarfs. Studies of the secondary eclipses of M-dwarfs around larger FGK stars in the infrared have found temperatures much hotter than evolutionary models predict (Triaud et al. 2013; Torres 2013). Empirical measurements of radius share a similar issue, in that they are typically underestimated by at least 3\% (Gómez Maqueo Chew et al. 2014; Demory et al. 2009; Spada et al. 2013; Fernandez et al. 2009; Nefs et al. 2013). The favoured explanation is that enhanced magnetic activity in M-dwarf stars inhibits the convective flow of energy to the surface, resulting in an enlarged radius (Morales et al. 2010; Fernandez et al. 2009; Zhou et al. 2014; Kraus et al. 2011). Many measurements of mass and radius are done from eclipsing binary systems, and some have suggested that tidal interactions (via rapid rotation) are the root of the problem (Kervella et al. 2016). However, there is evidence for radius inflation in single stars (Spada et al. 2013) suggesting a real problem with evolutionary models.

M-dwarfs are becoming prime targets for transiting exoplanet surveys. With all else constant, the transit of an exoplanet in 
the habitable zone is more likely to be observed around a star of lower mass to produce deeper eclipses that enable planetary atmospheric studies with transmission spectroscopy (Sedaghati et al. 2016; Southworth et al. 2017). Exciting new discoveries such as the TRAPPIST-1 system (Gillon et al. 2017) and Proxima Centauri b (Anglada-Escudé et al. 2016) have attracted attention from the scientific community and public alike, bringing more focus to low-mass stars in exoplanet surveys.

We are presented with an opportunity to use EBLMs discovered by the WASP survey to measure the properties of M-dwarfs. Measurements of $T_{\text {eff }}$ and $[\mathrm{Fe} / \mathrm{H}]$ for the FGK star can be measured accurately from a well understood spectrum and can be combined with radial-velocity measurements and transit photometry to obtain the mass, radius, and temperature of both components in EBLM systems. Measurements of $[\mathrm{Fe} / \mathrm{H}]$ for the M-dwarf can be adopted from the FGK companion with the assumption that they both formed from the same parent molecular cloud (Triaud et al. 2011).

The EBLM project (Triaud et al. 2013; Gómez Maqueo Chew et al. 2014; von Boetticher et al. 2017) is an ongoing effort to characterise transiting M-dwarfs in EBLM systems. Accurate estimates for temperature and composition are needed to estimate limb-darkening coefficients and make mass estimates for the primary star. For EBLM systems discovered by WASP, these parameters are made with CORALIE spectra using measurements of equivalent widths (EWs) and by fitting individual spectral lines (Gillon et al. 2009; Doyle et al. 2013; Doyle 2015). The individual analysis of each spectrum is acceptable for a small sample size, but we require a reliable automated procedure to measure $T_{\mathrm{eff}},[\mathrm{Fe} / \mathrm{H}]$, and $V \sin i$ for the entirety of the EBLM database to keep pace with future EBLM discoveries. At the same time, such a method needs to allow for noise and systematic errors present in the CORALIE spectra. The sample of EBLM spectra presented in Gómez Maqueo Chew et al. (2014) typicaly have a signal-to-noise ratio (S/N) per Ångstrom of between 3 and 7. The on-going radial velocity campaign to study EBLMs typically yields between 10 and 40 spectra per star. Co-adding spectra can increase the $\mathrm{S} / \mathrm{N}\left(\propto \sqrt{N_{\text {obs }}}\right)$ to over 40 in some parts of the spectrum, but the regions of the spectrum near the ends of each echelle order suffer from both large photon noise and systematic errors due to inaccurate order-merging.

Wavelet decomposition has been used previously as part of methods developed for spectral analysis. Manteiga et al. (2010) used multi-level wavelet decomposition in connectionist systems (artificial neural networks) to derive fundamental stellar parameters in the low-S/N domain (5-25) in preparation for spectra from the Gaia radial velocity spectrograph (RVS). This work was extended by Dafonte et al. (2016) by using a generative artificial neural network resulting in predicted uncertainties of $220 \mathrm{~K}$, 0.32 dex and 0.20 dex for $T_{\text {eff }}, \log g$ and $[\mathrm{Fe} / \mathrm{H}]$, respectively for stars with a Gaia magnitude $G_{\mathrm{RVS}}=13$. Using neural networks to estimate atmospheric properties entails well-known problems such as long training times and a strong dependence on the initial training set. $\mathrm{Li}$ et al. (2015) use wavelet decomposition in a regression framework to detect representative spectral features from a spectrum and estimate atmospheric parameters with better precision than those from neural networks $(83 \mathrm{~K}, 0.23 \mathrm{dex}$ and $0.16 \mathrm{dex}$ for $T_{\mathrm{eff}}, \log g$ and $\left.[\mathrm{Fe} / \mathrm{H}]\right)$.

Our method determines the best-fitting atmospheric parameters $\left(T_{\text {eff }},[\mathrm{Fe} / \mathrm{H}], V \sin i\right.$ and $\left.\log g\right)$ for FGK stars by comparing a selected subset of coefficients from a wavelet decomposition to those from a grid of stellar models. This reduces systematic errors in the estimated parameters due to poor continuum normalisation and low-quality regions of the spectrum. These measurements can then be combined with photometric followup observations to obtain the mass and radius of $\mathrm{M}$-dwarfs in EBLMs to an accuracy of a few percent. These, in turn, provide calibratable points for empirical mass-radius relations of low-mass stars (e.g. Demory et al. 2009; Torres et al. 2010). We introduce wavelet decomposition as it applies to a spectrum in Sect. 2 before reviewing our Bayesian approach to determine $T_{\text {eff }},[\mathrm{Fe} / \mathrm{H}], \log g$ and $V \sin i$ in Sect. 3. We show that our method converges and is self-consistent in Sect. 4 and test against a sample of independently analysed FGK stars in Sect. 5.

\section{Wavelet analysis}

Analysis of spectral components at different scales can be done using a discrete wavelet transform (DWT). A DWT tiles the wavelength-scale plane by convolving a spectrum, $f(\lambda)$, with variable sized functions (Stumpe et al. 2012). These functions are called daughter wavelets, $\psi_{\mathrm{a}, \mathrm{b}}(\lambda)$, which are created from a mother wavelet, $\psi(\lambda)$, using a shift-and-scale operation,

$\psi_{a, b}(\lambda)=\frac{1}{\sqrt{a}} \psi\left(\frac{\lambda-b}{a}\right), \quad a, b \in \mathfrak{R}, a \neq 0$,

where $a$ is a member of the dyadic sequence,

$a_{i}=2^{i}, \quad i=0,1,2,3, \ldots, n$

and $b=k b_{0}$, where $k$ is an integer and $b_{0}$ is chosen to ensure the recovery of $f(\lambda)$. By employing a DWT, the appropriate values of $b$ are selected to minimise overlap between wavelet convolutions. Following the notation in Chapter 8 of Olkkonen (2011), a discrete wavelet transform can be calculated for each dyadic scale $(i)$ and displacement $(k)$ :

$$
W T_{f(\lambda)}(i, k)=\frac{1}{\sqrt{2^{i}}} \int f(\lambda) \overline{\psi\left(\frac{\lambda-k 2^{i} b_{0}}{2^{i}}\right)} \mathrm{d} \lambda=\left\langle f(\lambda), \psi_{i, k}(\lambda)\right\rangle .
$$

The likeness of a wavelet, $\psi_{i, k}$, to a section of the spectrum is given by the wavelet coefficient $W T_{f(\lambda)}(i, k)$ from Eq. (3). Performing this calculation over the series of dyadic scales and displacements yields wavelet coefficients which represent different sized structures at different wavelengths. We split coefficients into bands with constant scales, $\left\{W T_{f(\lambda)}(0, b)\right\}_{k}$, which represent the likeness of a single scale across the entire spectrum. The power of each scale, $\left\{W T_{f(\lambda)}(i, b)\right\}_{k}^{2}$, can be visualised in a power Hövmoller (one value of $i$ per row) in Fig. 1. Bands of coefficients which correspond to noise and low-order continuum artefacts (such as merged échelle orders) can then be excluded. A filtered spectrum may be reconstructed with an inverse DWT (IDWT):

$f(\lambda)=\sum_{i=-\infty}^{\infty} 2^{\frac{-3 i}{2}} \int W T_{f(\lambda)}(i, k) \hat{\psi}\left(\frac{\lambda-b}{2^{i}}\right) \mathrm{d} b$,

where

$\hat{\psi}\left(\frac{\lambda-b}{2^{i}}\right)=\frac{\psi\left(\frac{\lambda-b}{2^{i}}\right)}{\sum_{i=0}^{i=n}|\psi(\lambda-b)|^{2}}$.

The process of reconstructing a spectrum using a subset of wavelet coefficients is called wavelet filtering and is analogous with Fourier filtering. Alternatively, the subset 


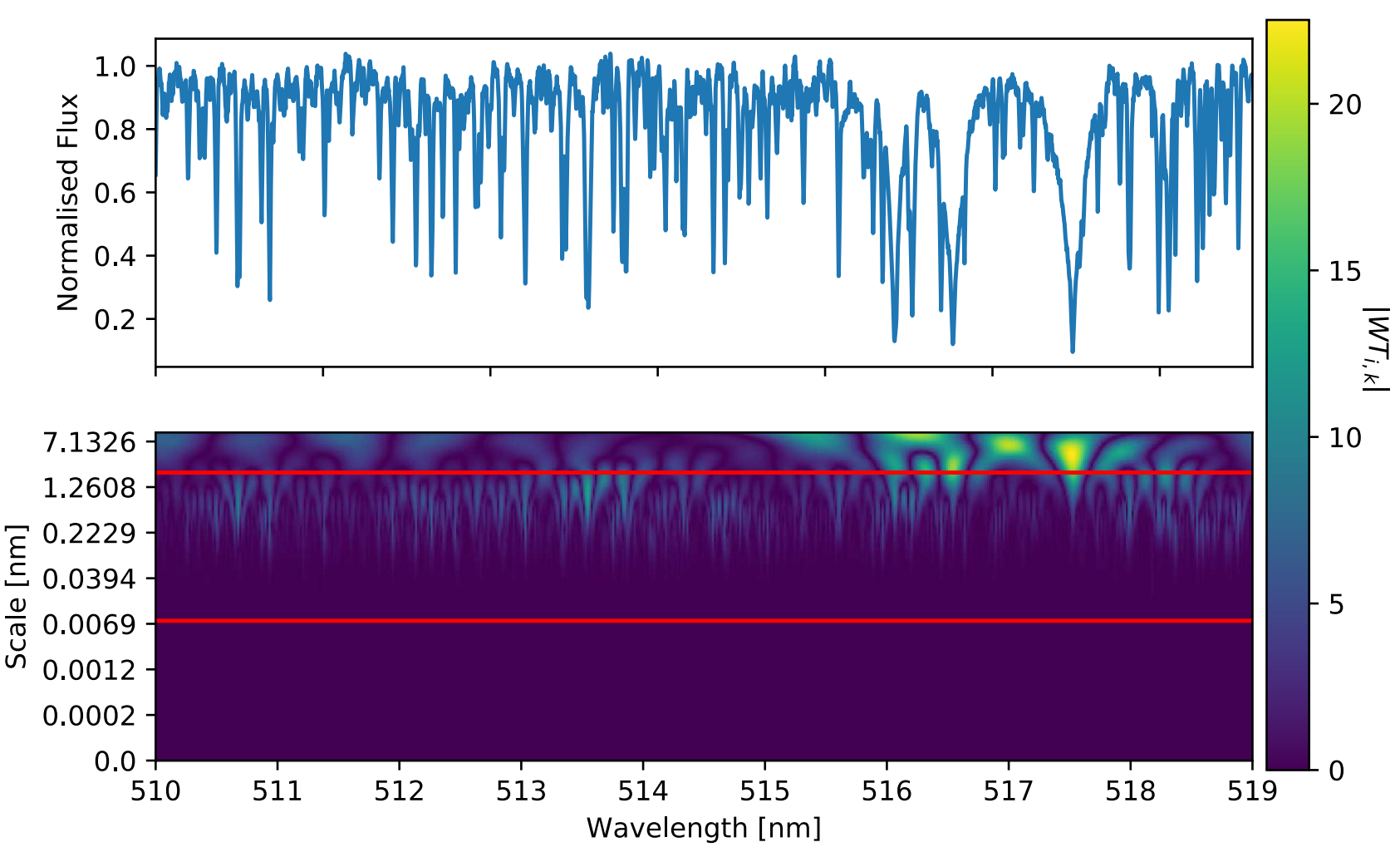

Fig. 1. Power Hövmoller of wavelet coefficients (lower panel) for a region around the Mg triplet for WASP-19 (upper panel). There is significant power $\left(\left|W T_{i, k}\right|\right.$ from Eq. 3) for scales $\sim 1 \mathrm{~nm}$ in the region of the $\mathrm{Mg}$ lines corresponding the wavelets likeness to spectral features. Horizontal red lines represent the scales $0.012-3.125 \mathrm{~nm}$.

of coefficients may be chosen to meet a threshold criteria (i.e. $\left.\left[W T_{f(\lambda)}(i, k)\right]^{2} \geq 0.01\right)$ which eliminates information that has little contribution to a signal; this is called wavelet compression.

We do not require Eq. (4) to determine atmospheric parameters as we perform a $\chi^{2}$ fit using a subset of coefficients from Eq. (3) to those from a grid of models (see Sect. 3). We also do not apply any threshold criterion. The nominal resolving power of the CORALIE spectrograph is $R=55000$, so at least $2^{16}$ values are required to sample a spectrum over the wavelength range $450-650 \mathrm{~nm}$. We decided to use $2^{17}$ values for the wavelet decomposition to ensure no loss of information and to give us more choice in the number of wavelet bands used in our analysis. We use Eq. (3) to obtain wavelet coefficients which have information on scales in the range $0.003 \mathrm{~nm}-200 \mathrm{~nm}$. Our wavelet method only uses a subset of $i$ values. To select these, we constructed power Hövmoller diagrams (similar to Fig. 1) for a variety of regions between $450 \mathrm{~nm}$ and $650 \mathrm{~nm}$, for different co-added spectra in our sample. We found that power associated with line absorption lies in the range $0.04-4 \mathrm{~nm}$, with larger scales typically corresponding to systematic trends and shorter scales with noise. This corresponds to values of $i=4-12$ $(0.048-3.125 \mathrm{~nm})$. The application of Eq. (4) to the two subsets of coefficients (4-12 and 13-17) is shown in Fig. 2a. We find that the subset range $i=4-12$ is too restrictive to reproduce short-scale information (e.g., weak lines) and so we decide to extend this range to $i=4-14(0.012-3.125 \mathrm{~nm}$; Fig. 2b) which better represents the boundary between noise and weak lines. We do not show the reconstruction of subset $i=0-3$ in Figs. 2a and $\mathrm{b}$ as using only 16 coefficients to reconstruct a spectrum leads to a large Daubechies-4 wavelet with some sub-structure.

We demonstrate the sensitivity of wavelet coefficients to atmospheric parameters in Fig. 3 for wavelet coefficients in the range $i=11-12(0.04-0.09 \mathrm{~nm})$. We see a slow variation of some wavelet coefficients which corresponds to changes in individual spectral line geometries as each parameter changes. One thing to note is the sensitivity of each parameter; $T_{\text {eff }}$ varies the most, followed by $V \sin i$ and $[\mathrm{Fe} / \mathrm{H}]$. Surface gravity is the least varying parameter in wavelet space and is dominated by a few lines sensitive to $\log g$. As $V \sin i$ increases, we see positive and negative structures form and become stronger at higher $V \sin i$ values. This is likely to be a continuum effect as weaker lines are smeared out to average a lower continuum whilst stronger lines persist.

The choice of mother wavelet depends on the objective of the work. A Daubechies wavelet performs well for frequency identification and is widely used in signal processing and data compression (e.g. Belmon et al. 2002). A Haar wavelet, with a more step-like structure, is more suited to identifying discontinuity and is widely used in computer-vision projects (e.g. Essaouabi et al. 2009). We investigate the effects of wavelet choice on the determined atmospheric parameters in Sect. 5.2.1, but proceed with the Daubechies $(k=4)$ wavelet for the rest of this paper.

\section{Bayesian measurements}

We use the Markov chain Monte Carlo method to determine the posterior probability distribution for $T_{\mathrm{eff}}, \log g, V \sin i$ and $[\mathrm{Fe} / \mathrm{H}]$ given an observed spectrum. Our method is a global $\chi^{2}$ fitting routine which compares subsets of wavelet coefficients $(i=4-14)$ to those from a pre-synthesised grid of spectra. Our grid was synthesised with the radiative transfer code SPECTRUM (Gray \& Corbally 1994) using MARCS model atmospheres (Gustafsson et al. 2008), and version 5 of 


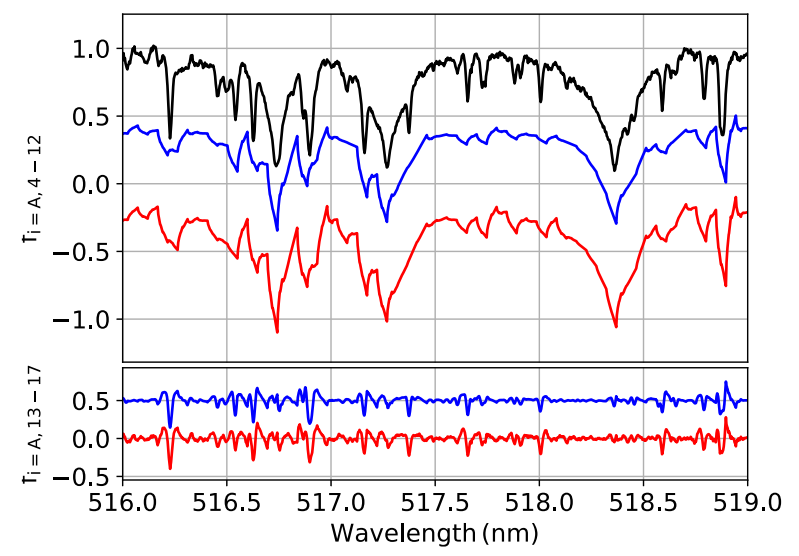

(a)

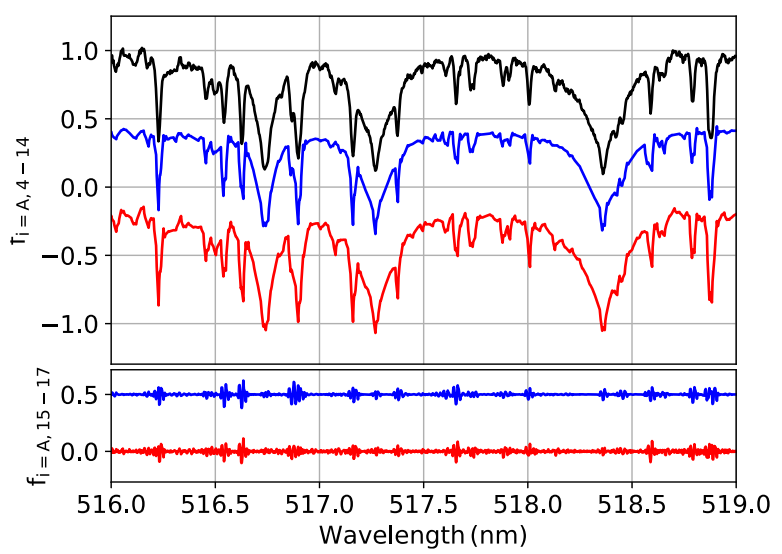

(b)

Fig. 2. Reconstruction of spectra using Eq. (4) for subsets of wavelet coefficients. (Left panel - top) Raw spectra for WASP-19 (black) and the flux reconstruction using wavelet coefficients from bands $i=4-12$ using the raw spectrum (blue; offset -0.6$)$ and the best fitting model for WASP-19 (red; offset -1.2). (Left panel - bottom) The reconstruction of the best-fitting model for WASP-19 (red) and the raw spectrum (blue; offset +0.5 ) using coefficients $i=13-17$. (Right panel) As in the left panel except with reconstructions using coefficients $i=4-14$ (top) and coefficients $i=15-17$ (bottom).

the GES (Gaia ESO survey) atomic line list provided within iSpec (Blanco-Cuaresma et al. 2016) with solar abundances from Asplund et al. (2009). We computed models spanning $450-650 \mathrm{~nm}$ over a temperature range of 4000 to $8000 \mathrm{~K}$ in steps of $250 \mathrm{~K},-1$ to +1 dex in steps of 0.5 dex for $[\mathrm{Fe} / \mathrm{H}]$ and 3.5 to 5 dex in steps of 0.5 for $\log g$. We selected our range of $[\mathrm{Fe} / \mathrm{H}]$ by looking at composition measurements of over 14000 FGK stars from Gaia-ESO survey data release 3 (Fig. 4; Smiljanic et al. 2014). We find that $96 \%$ of stars with measurements of composition had $[\mathrm{Fe} / \mathrm{H}]$ in the range -1 to $1 \mathrm{dex}$. This range in $[\mathrm{Fe} / \mathrm{H}]$ is also much larger than the full range in $[\mathrm{Fe} / \mathrm{H}]$ for our benchmark sample described in Sect. 5.

Spectra in the grid are calculated with zero instrumental, rotational, or macroturbulence broadening. These are accounted for in post-processing by convolving the grid spectra with the appropriate kernels. In this work, we allow $V \sin i$ to have values in the range $0-50 \mathrm{~km} \mathrm{~s}^{-1}$. The upper limit of $50 \mathrm{~km} \mathrm{~s}^{-1}$ would need to be extended for hotter stars beyond the Kraft break, but is suitable for this work on late-type stars. Macroturbulence are estimated using Eq. (5.10) from Doyle (2015) and microturbulence was accounted for at the synthesis stage using Eq. (3.1) from the same source. Spectra in-between grid points are extracted by trilinear interpolation, broadened to the desired value of $V \sin i$ and macroturbulence, and then convolved with a Gaussian to account for instrumental broadening. For the self-consistency tests in Sect. 4 instrumental broadening was ignored, but for the CORALIE spectra in Sect. 5 we used an instrumental resolving power $R=55000$ (Queloz et al. 2001; Doyle 2015). We then re-sample between 450 and $650 \mathrm{~nm}$ with $2^{17}$ values (the same as the observed spectrum) and apply Eq. (3) to obtain the wavelet coefficients $W T_{f(\lambda)}(4-14, k)$ for the model spectra.

The subset of wavelet coefficients from the interpolated model, $W T_{\mathbf{m}}$, are compared to those from the data, $W T_{\mathbf{d}}$, in the following Bayesian framework: the probability of observing a spectrum for a given model is given by $\mathrm{p}(\mathbf{m} \mid \mathbf{d}) \propto$ $\mathcal{L}(\mathbf{d} \mid \mathbf{m}) \mathrm{p}(\mathbf{m})$. The vector of model parameters is given by $\mathbf{m}=\left(T_{\text {eff }},[\mathrm{Fe} / \mathrm{H}], \log g, V \sin \mathrm{i}\right)$ and we assume uniform prior probability for the model parameters within the grid range. We use the likelihood function $\mathcal{L}(\mathbf{d} \mid \mathbf{m})=\exp \left(-\chi^{2} / 2\right)$ where

$\chi^{2}=\frac{\left(W T_{\mathbf{d}}-W T_{\mathbf{m}}\right)^{2}}{\sigma_{W T_{\mathbf{d}}}^{2}}$,

and

$\sigma_{W T_{\mathbf{d}}}^{2}=\beta \sigma_{M C}^{2}$.

The term $\sigma_{M C}^{2}$ was calculated by generating 1000 spectra from the co-added spectrum with noise generated from a standard normal distribution centred around $f(\lambda)$ and with $\sigma$ equal to the standard deviation of the spectrum, $\sigma_{f(\lambda)}$ (calculated from the standard deviation in co-added spectra). The blaze function is corrected prior to co-addition of the spectra and so deviations in blaze functions will result in uncertainties propagating through to $\sigma_{f(\lambda)}$, and $\sigma_{M C}^{2}$, effectively down-weighting regions with poor blaze corrections. The free parameter $\beta$ has been introduced to account for additional noise, incomplete atomic data, deviations from solar metallicity scaling, lines which form under non-local thermodynamic equilibrium, and other unaccounted errors. In principle, we could have used stellar models or empirical relations to set priors on these atmospheric parameters but decided not to do this for two reasons. Firstly, allowing the MCMC sampler to explore regions with a priori low probability gives a better indication of the reliability of our method than using a more constrained solution. Secondly, by imposing a prior from stellar models or empirical relations based on normal stars we may fail to identify interesting examples of anomolous stars in our sample, for example, helium-rich stars.

We sample the model parameter space using the Markov chain Monte Carlo method, implemented by the python package EMCEE (Foreman-Mackey et al. 2013). EMCEE uses affine-invariant ensemble sampling (Goodman \& Weare 2010, parallel stretch move algorithm) to split Markov chains into sub-groups and update the position of a chain using the positions of chains in the other subgroups. The algorithms affine-invariance can cope with skewed probability distributions and generally has shorter autocorrelation times than a classic Metropolis-Hastings algorithm. 

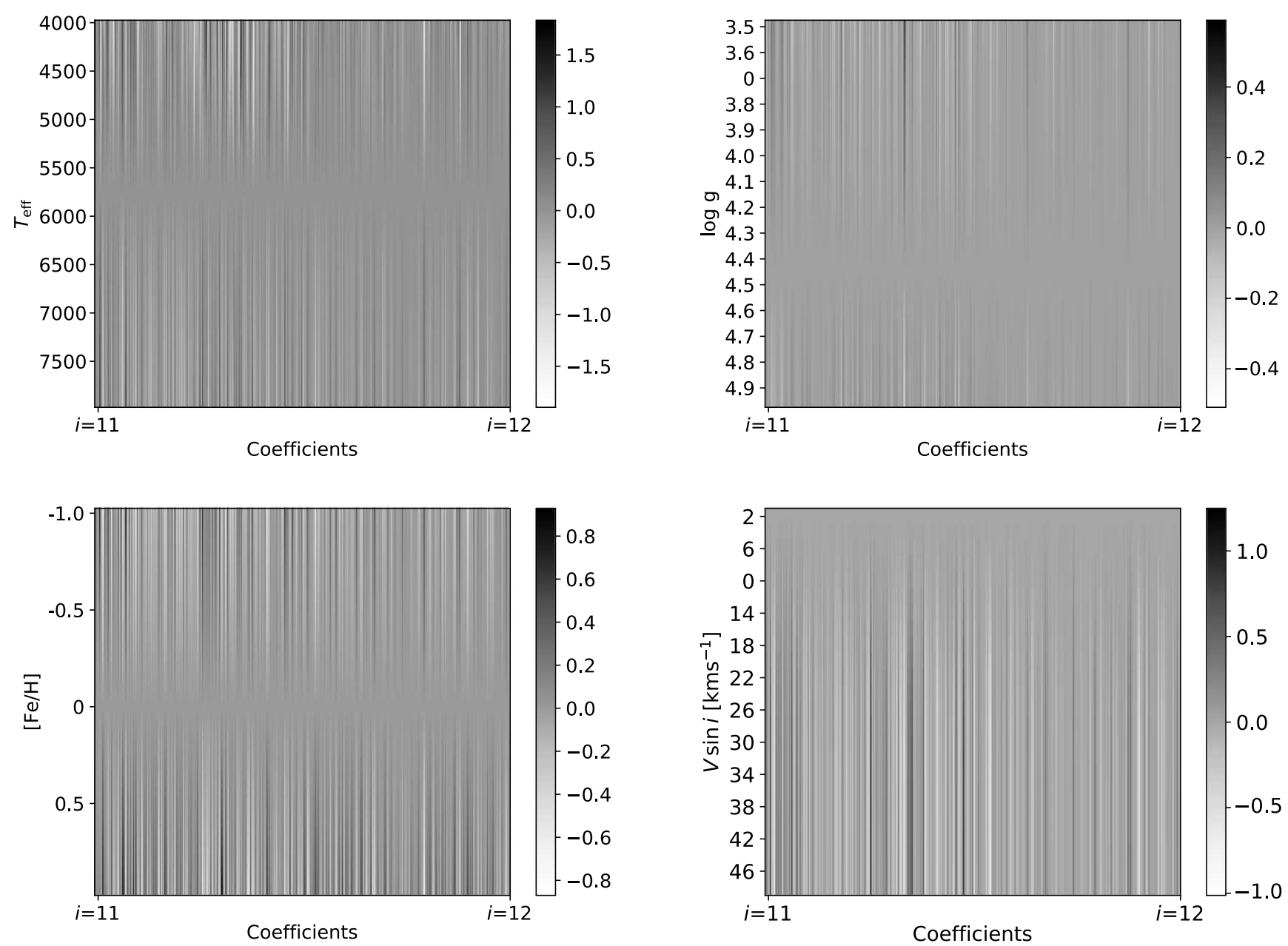

Fig. 3. Changes in wavelet coefficients in the range $i=11-12$ for model spectra as a function of atmospheric parameters. The wavelet coefficient from a solar model has been subtracted to emphasise the subtle change in wavelet coefficients for each parameter. A similar result is seen for other values of $i$ between 4 and 14. The colour-bar indicates the magnitude of the difference of coefficients. We note that these bars are not on the same scale and highlight the wavelet response to each coefficient.

We generate 12 Markov chains of 20000 draws each to converge on the best atmospheric parameters. We found that the chains converged before the 5000 th draw, but as a precaution we discarded the first 10000 draws. We take the median values of the model parameters in the remaining draws to determine the atmospheric parameters for a spectrum. An example posterior probability distribution for WASP-20 is plotted in Fig. 5. The parameter space is almost symmetric with small degeneracies between $T_{\text {eff }},[\mathrm{Fe} / \mathrm{H}]$ and $\log g$. We note that the precision of the parameters determined from the standard deviation of each parameter in the Markov Chain is typically an underestimate of the true precision of these parameters because it does not account for systematic errors in the data or the models.

\section{Self consistency}

We have assessed the ability of our method to recover atmospheric parameters from synthetic spectra in order to check that our results are self consistent. We interpolated 512 spectra with random values of $T_{\text {eff }},[\mathrm{Fe} / \mathrm{H}], \log g$ and $V \sin i$ selected within the limits of our grid of models. Each spectrum was then re-sampled to have $2^{17}$ values in the range $450-650 \mathrm{~nm}$ to match our choice of coefficients in Sect. 2 and the benchmark sample in Sect. 5. These spectra were split into two groups and analysed with the aforementioned method. The first group had $\log g$ as a free parameter to probe for any systematics, for the second group we imposed a prior on $\log g$ to simulate the effect of well constrained surface gravity measurement from transit photometry. The $\log g$ prior probability distribution was assumed to be Gaussian with a mean $\log g$ value equal to the value used to interpolate the spectrum and a dispersion equal to the average uncertainty of transit $\log g$ values from Mortier et al. (2013, hereafter referred to as M13) for 44 WASP exoplanet hosts $\left(\overline{\sigma_{\log g}}=\right.$ 0.02 dex). We decided not to add Gaussian noise to these spectra as noise profiles depend upon stellar parameters and instrumental conditions; this is assessed in Sect. 5.2.4. We find typical autocorrelation lengths are below 1000 steps for all parameters in the first chain and 12 chains in the second run typically produce an acceptance fraction between $\sim 0.25$ and 0.3 .

The recovery of atmospheric parameters for both groups is shown in Fig. 6 and summarised in Table 1. We find that all parameters are recovered well across the range of our grid. With no constraints on $\log g$, there were only two measurements of $T_{\text {eff }}$ that deviated from the input value by more than $150 \mathrm{~K}$. A prior on $\log g$ significantly decreases the difference between measured and input atmospheric parameters and shows that our method is sensitve to $\log g$. There is a small increase in residual scatter for measurements of $V \sin i$ when the interpolated value of $V \sin i$ below $0.5 \mathrm{~km} \mathrm{~s}^{-1}$; this is seen in both groups and marginally improved with a prior on $\log g$. This is expected 


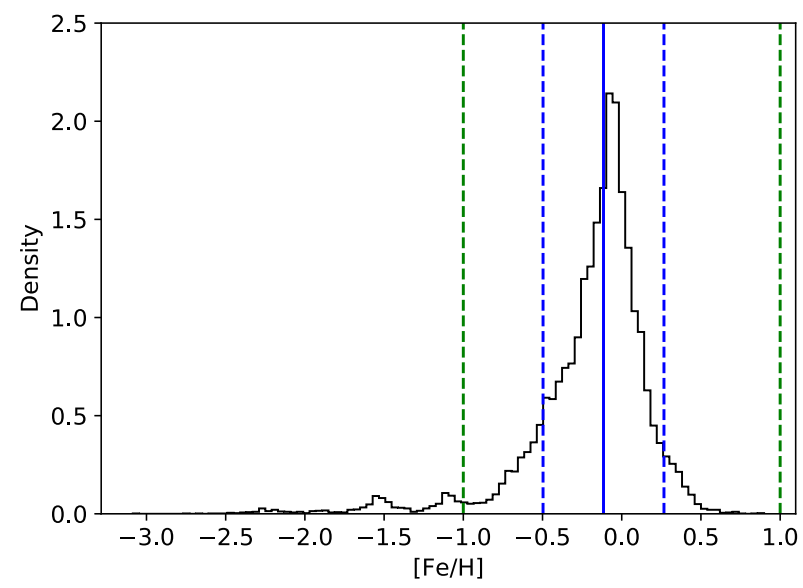

Fig. 4. Histogram of 14,681 [Fe/H] measurements for stars from GaiaESO data release 3 (Smiljanic et al. 2014). Plotted is the median value of $[\mathrm{Fe} / \mathrm{H}]$ (solid blue), with $1 \sigma$ from the median (dashed blue). The grid range used in enclosed by the dashed green lines.

Table 1. Recovery of atmospheric parameters using the wavelet method for two groups of 256 spectra: one group with no priors on $\log g$ and another with priors imposed from transit photometry.

\begin{tabular}{lccc}
\hline \hline & $\begin{array}{c}\text { Prior on } \\
\log g ?\end{array}$ & $\sigma$ & $\mu$ \\
\hline$T_{\text {eff }}(\mathrm{K})$ & no & 46.0 & -3.2 \\
& yes & 3.1 & 0.2 \\
{$[\mathrm{Fe} / \mathrm{H}](\mathrm{dex})$} & no & 0.040 & -0.003 \\
& yes & 0.020 & -0.001 \\
$V \sin i\left(\mathrm{kms}^{-1}\right)$ & no & 0.47 & 0.05 \\
& yes & 0.17 & -0.06 \\
$\log g(\mathrm{dex})$ & no & 0.060 & -0.002 \\
& yes & 0.020 & 0.001 \\
\hline
\end{tabular}

Notes. The difference between the value measured by the wavelet method and the input value used to interpolate the spectrum $\left(x_{\text {out }}-x_{\text {in }}\right)$ were used to calculate the standard deviation, $\sigma$, and mean offset, $\mu$.

as the resolution of the broadening kernel in combination with the edge of parameter space makes it difficult to determine low $V \sin i$ values. The internal precision associated with the wavelet method is remarkably high; by taking $1 \sigma$ values from the cumulative probability distributions we find precisions around $15 \mathrm{~K}$, $0.01 \mathrm{dex}, 0.02 \mathrm{dex}$, and $0.15 \mathrm{~km} \mathrm{~s}^{-1}$ for $T_{\text {eff }},[\mathrm{Fe} / \mathrm{H}], \log g$, and $V \sin i$ respectively. More realistic uncertainties are determined in the following sections.

We also assess the sensitivity of our method by determining the atmospheric parameters of 9 spectra from a discrete set of grid points with different combinations of fixed parameters. We interpolate 96 spectra from the following grid points $4800 \mathrm{~K}, 5800 \mathrm{~K}$ and $6500 \mathrm{~K}$ for $T_{\text {eff }} ;-0.5,0.0$ and 0.5 dex for $[\mathrm{Fe} / \mathrm{H}] ; 3.75,4.40$ and 4.80 dex for $\log g ; 5,10$ and $15 \mathrm{kms}^{-1}$ for $V \sin i$. In total, we determined the atmospheric parameters for each spectrum 15 times with the wavelet method using every combination of free and fixed parameters (see Fig. 7). We find that constraining one or more parameters increases the internal precision of the wavelet method significantly. A slight degeneracy exists between $T_{\text {eff }}$ and $\log g$ resulting in a modest scatter when both parameters are left free. This also highlights the numerical noise introduced by starting walkers at different positions, since walkers explore parameter space by random jumps which may never reach the correct solution, despite prior knowledge that an exact solution lies somewhere within the grid.

\section{Benchmark sample}

Any spectral analysis technique must be tested against stars with high-quality measurements. For this we use stars from Doyle et al. (2013) and Doyle (2015, D15, hereafter). The D15 sample consists of 24 stars analysed by measurements of EW and spectral fitting of high-S/N and high-resolution $(R=112000)$ data from the HARPS spectrograph (Queloz et al. 2001). We used lower-quality observations from the CORALIE spectrograph to determine $T_{\text {eff }},[\mathrm{Fe} / \mathrm{H}], \log g$ and $V \sin i$ of the same stars with the wavelet method. Only 22 stars in the D15 sample have CORALIE spectra available to use and we further exclude WASP-77A and the close ( $\left.3^{\prime \prime}\right)$ B-component as both component as they are un-resolved in the CORALIE fibre. This leaves a sample of 20 stars for use to calibrate our method (see Table 2).

\subsection{CORALIE spectra}

Each spectrum was processed with the CORALIE standard datareduction pipeline (Baranne et al. 1996). The radial velocity shift was measured relative to a solar template ${ }^{2}$ and corrected into a laboratory frame of reference. The spectra were then median-combined onto an identically sampled wavelength grid. Continuum regions were identified by applying maximum and median filters (Blanco-Cuaresma et al. 2014) and fitted with spline functions ( 1 every $10 \mathrm{~nm}$ ) for normalisation. The wavelet method was then applied to each spectrum twice: once with no priors on $\log g$ and a second time with priors given by transit photometry. The priors on $\log g$ were set to those from M13 if quoted, or the relevant discovery papers otherwise (see Table 6).

\subsection{Results}

The results can be seen in Fig. 8 and are summarised in Tables 3 and 6. Our method determines $T_{\text {eff }}$ to within $220 \mathrm{~K}$ of the value found by $\mathrm{D} 15$. Our measurements of $[\mathrm{Fe} / \mathrm{H}]$ are systematically offset by approximately -0.18 dex from those of D15; this is discussed further in Sect. 5.2.1. It is difficult to constrain $\log g$ spectroscopically and our measurements often differ from those of D15 by up to 0.5 dex. Our measurements of $V \sin i$ converge to $0 \mathrm{~km} \mathrm{~s}^{-1}$ for seven stars in the sample due to an over estimation of $v_{\text {mac }}$ or instrumental resolution. To mitigate this problem we repeated the analysis with $v_{\mathrm{mac}}=0 \mathrm{~km} \mathrm{~s}^{-1}$. This allowed these stars to converge on best fitting models without pushing against the edge of parameter space. These stars are marked with an asterisk in Table 6.

We find no benefit by using priors on $\log g$. In most cases the use of $\log g$ priors increases the standard deviation in differences between atmospheric parameters from our method and published values. We investigated the level of agreement between spectroscopic values of $\log g$ from EW measurements (D15), $\log g$ from the wavelet method and those from transit photometry. Photometric surface gravity is typically measured to better precision than its spectroscopic counterpart, but relies on stellar models and correct limb-darkening parameters which in-turn rely on a constrained effective temperature, composition, and surface gravity. Recent work suggests a disagreement between spectroscopic and photometric $\log g$ which is correlated

2 From the Gaia Benchmark Stars Library pipeline which is the result of co-adding asteroid observations by NARVAL. 


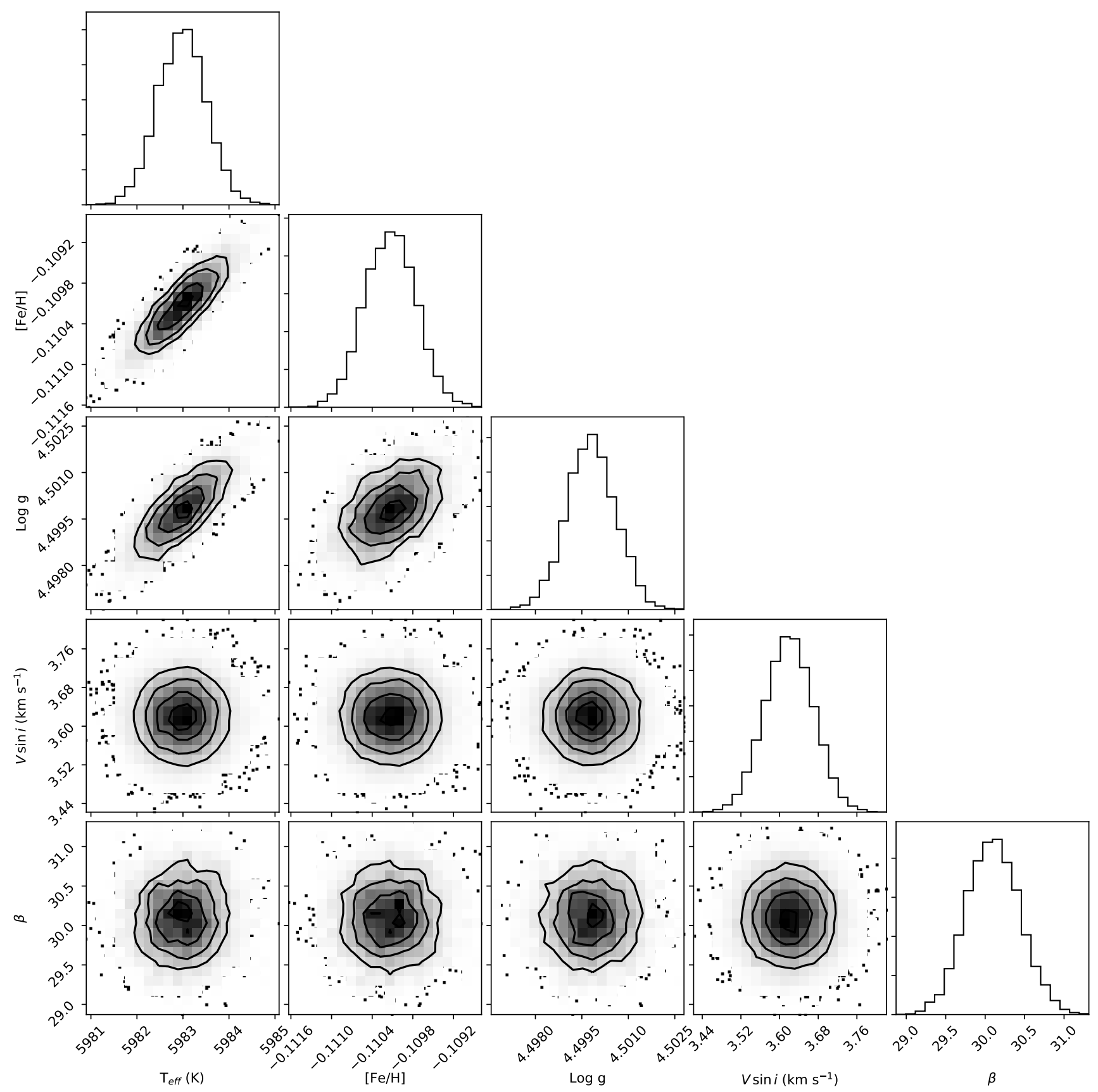

Fig. 5. Posterior proability distributions for WASP-20.

with $T_{\text {eff }}$ (see Fig. 4 from Doyle et al. 2017). We compare the difference between spectroscopic and photometric $\log g$ measurements in Fig. 9. We find a statistically significant negative correlation ( $\mathrm{p}$-value $\leq 10^{-5}$ ) between $\Delta \log g$ ( $\log g_{\text {photometry }}$ $\log g_{\text {wavelet }}$ ) with $T_{\text {eff }}$ from our method. The origin of this is unclear, but a similar trend is seen between spectroscopic and asteroseismic measurements (Fig. 6 of Mortier et al. 2014) which suggests to us that this is a problem to spectral analysis of latetype stars using plane-parallel non-LTE model atmospheres. For a few stars, we relaxed the $\log g$ prior to have a standard deviation of 0.2 dex (instead of $0.02 \mathrm{dex}$ ) and found almost no difference between these solutions and those with a uniform prior on $\log g$.

In Fig. 10 we assess the $\mathrm{H} \alpha$ region for the model predicted from D15 and this work for the highest-quality spectrum in our sample, WASP-20, with $S / N=150$. The results from D15 were obtained using a custom line list, whereas we use version 5 of the GES atomic line list provided with iSpec to synthesise the D15 model of WASP-20 using atmospheric parameters, $v_{\text {mac }}$ and $v_{\text {mic }}$ from D15. We find both models agree well with the data, with the left wing fitting best and a underestimation in the right wing. The discrepancies between the two wings of the $\mathrm{H} \alpha$ line seen here are the result of the difficulty in calibrating the blaze function in this region of the spectrum. We find that the majority of Fe line depths are under-predicted with the wavelet method, with the D15 model better matching individual line profiles. This test demonstrates the need to benchmark against well studied stars and visually inspect the best models against the data. 

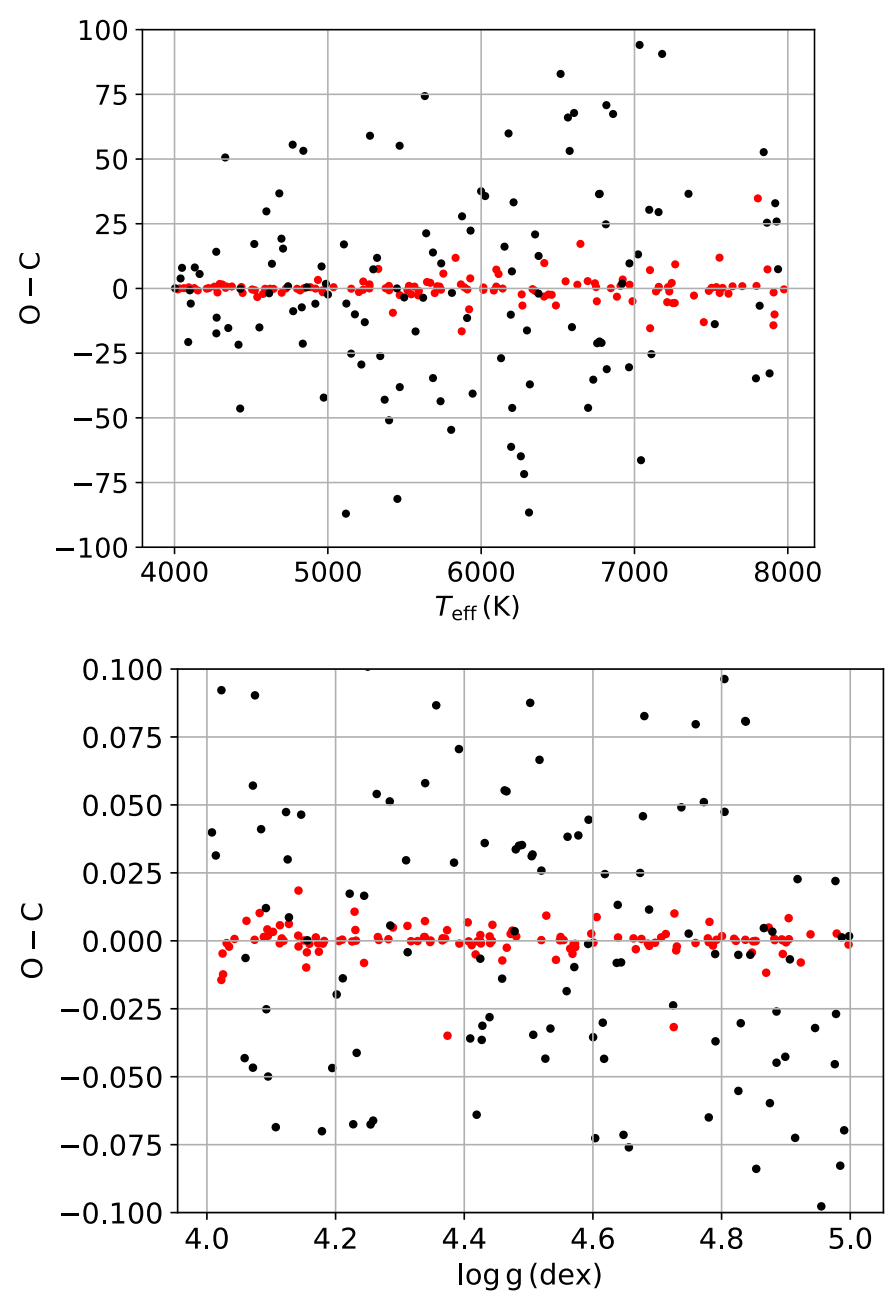
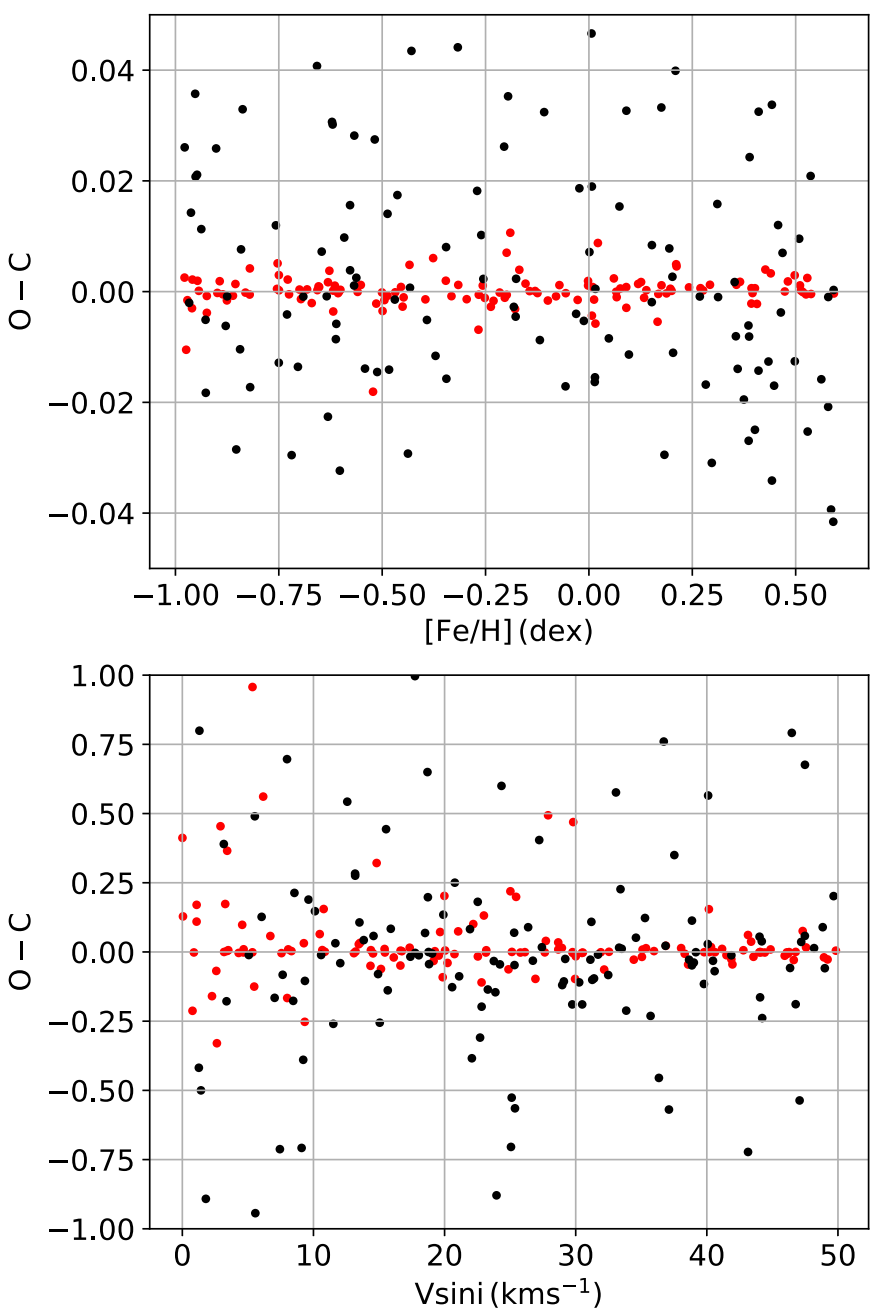

Fig. 6. Differences between wavelet-determined atmospheric parameters and those used to synthesize spectra with all parameters free (black) and with priors on $\log g$ (red).

\subsubsection{Systematic offset in $[\mathrm{Fe} / \mathrm{H}]$}

There are many reasons why our method may be produce composition offsets compared with other established techniques. The interested reader should see Jofré et al. (2017) for an excellent review on how the specifics of spectroscopic analysis routines affect abundance measurements. One interesting result from Jofré et al. (2017) is the effect of continuum normalisation which increased the method-to-method scatter in abundance measurements by up to 0.3 dex (see their Fig. 5). Wavelet filtering in our method is an alternate approach to normalisation, and so an offset of around $0.18 \mathrm{dex}$ is not entirely unexpected. We assess if there is a systematically lower continuum placement by adding an free parameter, $C_{0}$, which is a constant to add to the normalised flux of the model spectra before a discrete wavelet transform in the calculation of log-likelihood. We found values of $C_{0}$ converged to values between -0.05 and 0.05 and did not affect measurements of $[\mathrm{Fe} / \mathrm{H}]$ by more than $0.05 \mathrm{dex} ; T_{\text {eff }}$ remained the same for all stars within $150 \mathrm{~K}$ and $\log g$ changed by as much as 0.2 dex.

We also looked at components unique to the wavelet method. For instance, the mother wavelet used (Daubechies, $k=4$ ) may not capture the true line depths when convolved with a spectrum. We again measured WASP-20 with three alternative wavelets
(Daubechies $k=20$, Harr $k=2$ and bspline $k=103$ ) across the range 450-650 nm (see Table 4). We find the choice of mother wavelet has little influence on the determined composition (and all other atmospheric parameters) for WASP-20 and we find similar results for the rest of the D15 sample. It is possible that the resolution of the finest wavelet convolution ( 2 pixels) is not sufficient to capture iron line depths. To assess this, we convolved a few iron lines with the Daubechies $k=4$ kernel and assessed whether line depths were underdetermined. We found this not to be the case, suggesting no degradation of line depths owing to the choice in wavelets.

Finally, we consider the possibility that there may be instrumental effects at play with the CORALIE échelle spectrograph. A discrepancy in EW measurements for WASP-69 (see Fig. 3.19 in D15) suggests this instrument is prone to scattered light (Doyle 2015). This may be partly responsible for the systematic error in the iron abundance when combined with a low-quality spectrum.

The zero-point of the metallicity scale is a subject of ongoing debate (e.g. Kraft \& Ivans 2004). However, we can conclude that models using parameters found by D15 (as generated with line lists and atmospheres used in the above work) have better-fitting line depths for the majority of Fe lines in the D15 sample than our predicted models. For this reason, we advocate 
S. Gill et al.: The atmospheric parameters of FGK stars using wavelet analysis of CORALIE spectra
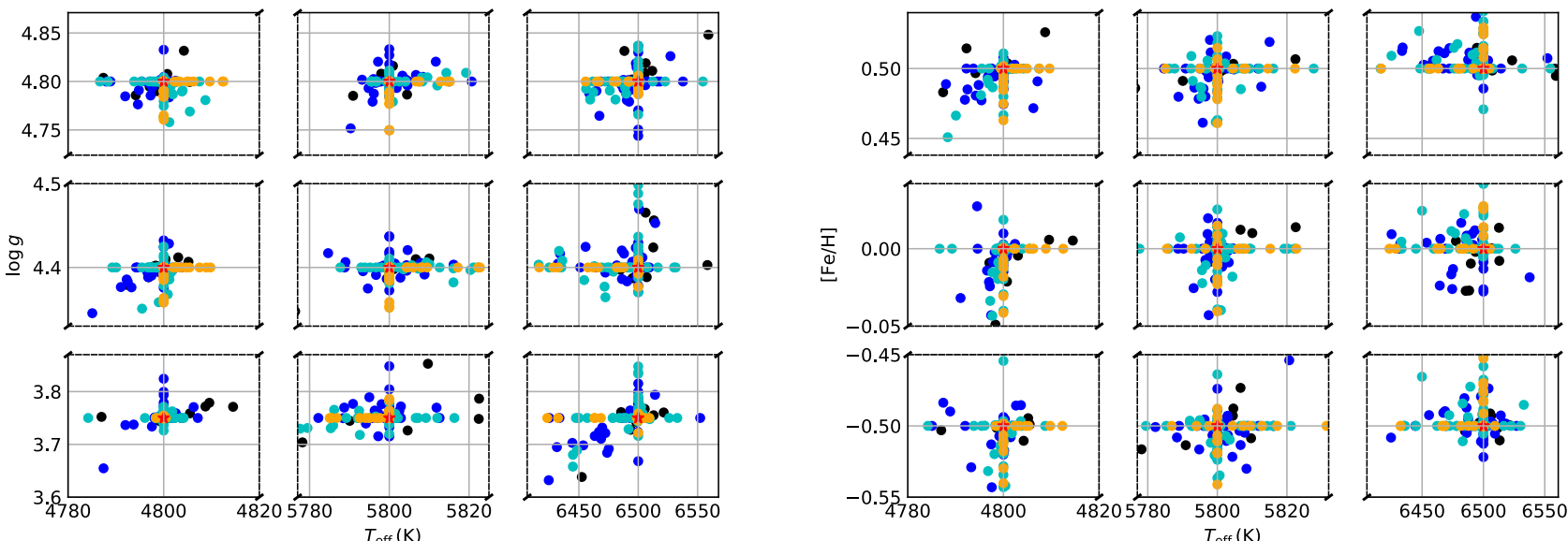

Fig. 7. Difference between atmospheric parameters determined by the wavelet method for 9 synthetic spectra when some parameters are fixed. We plot atmospheric parameters determined with with all parameters free $\left(T_{\text {eff }},[\mathrm{Fe} / \mathrm{H}], \log g, V \sin i\right)$ in black; with one parameter fixed in blue; with two parameters fixed in cyan; three parameters fixed in orange.

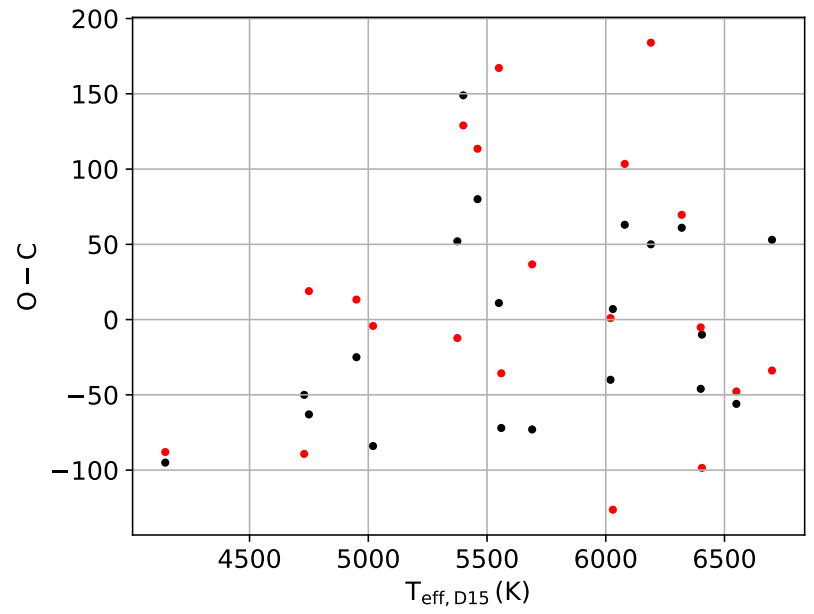

(a)

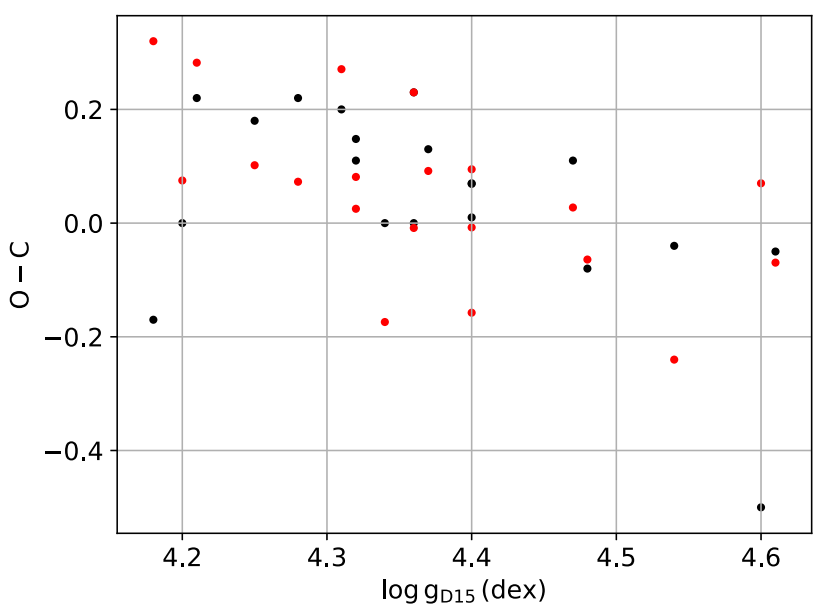

(c)

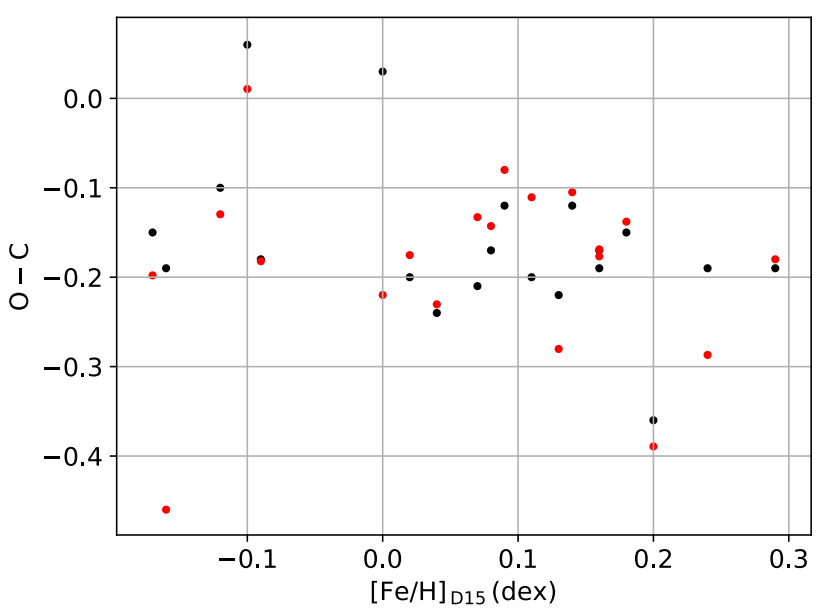

(b)

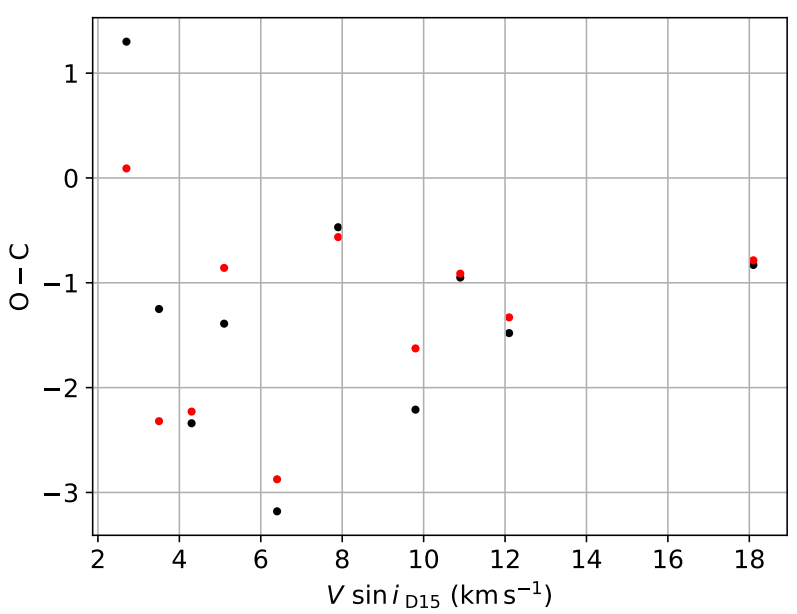

(d)

Fig. 8. Difference between wavelet analysis and D15 (O-C) for each atmospheric parameter in the D15 sample. Each spectrum was measured twice, once with $\log g$ as a free parameter (black) and again with $\log g$ priors imposed from transit photometry (red). We exclude measurements of $V$ sin $i$ where macroturbulence, $\xi_{t}$, was set to $0 \mathrm{~km} \mathrm{~s}^{-1}$ to ensure models a best model was converged upon. 
Table 2. Our benchmark sample of FGK stars from D15.

\begin{tabular}{lccc}
\hline \hline Star & $V$ mag & $\begin{array}{c}\text { \# of } \\
\text { spectra }\end{array}$ & $\begin{array}{c}S / N \\
(\sim 500 \mathrm{~nm})\end{array}$ \\
\hline WASP-4 & 12.50 & 12 & 37 \\
WASP-5 & 12.30 & 11 & 35 \\
WASP-6 & 11.90 & 30 & 63 \\
WASP-7 & 9.50 & 13 & 124 \\
WASP-8 & 9.79 & 21 & 137 \\
WASP-15 & 11.00 & 15 & 83 \\
WASP-16 & 11.30 & 19 & 77 \\
WASP-17 & 11.60 & 42 & 71 \\
WASP-18 & 9.30 & 5 & 119 \\
WASP-19 & 12.59 & 28 & 50 \\
WASP-20 & 10.68 & 58 & 153 \\
WASP-22 & 12.00 & 29 & 63 \\
WASP-23 & 12.68 & 38 & 53 \\
WASP-24 & 11.31 & 18 & 53 \\
WASP-29 & 11.30 & 14 & 57 \\
WASP-30 & 11.90 & 47 & 27 \\
WASP-31 & 11.70 & 35 & 53 \\
WASP-53 & 12.19 & 35 & 40 \\
WASP-69 & 9.88 & 21 & 136 \\
WASP-80 & 11.90 & 37 & 51 \\
\hline
\end{tabular}

Notes. We include the $V$ magnitude, the number of spectra and the $\mathrm{S} / \mathrm{N}$ of the coadded spectra at $500 \mathrm{~nm}$.

Table 3. Recovery of atmospheric parameters for 20 FGK stars from D15: one group with no priors on $\log g$ and another with priors from transit photometry.

\begin{tabular}{lccc}
\hline \hline & $\begin{array}{c}\text { Prior on } \\
\log g ?\end{array}$ & $\sigma$ & $\mu$ \\
\hline$T_{\text {eff }}(\mathrm{K})$ & no & 85.00 & 31.00 \\
& yes & 86.00 & 14.00 \\
{$[\mathrm{Fe} / \mathrm{H}](\mathrm{dex})$} & no & 0.06 & -0.15 \\
& yes & 0.10 & -0.18 \\
$V \sin i\left(\mathrm{~km} \mathrm{~s}^{-1}\right)$ & no & 1.35 & -0.79 \\
& yes & 0.62 & -1.33 \\
$\log g(\mathrm{dex})$ & no & 0.13 & 0.08 \\
& yes & 0.14 & 0.05 \\
\hline
\end{tabular}

Notes. The difference between the value measured by the wavelet method and D15 ( $\left.x_{\text {wavelet }}-x_{D 15}\right)$ are used to calculate the mean dispertion, $\sigma$, and mean offset $\mu$. Values of $\sigma$ and $\mu$ for $V \sin i$ excluded stars where macroturbulence, $\xi_{t}$, was set to $0 \mathrm{~km} \mathrm{~s}^{-1}$.

Table 4. Performance of the wavelet method using different mother wavelets.

\begin{tabular}{lcccc}
\hline \hline Wavelet & $\begin{array}{c}T_{\text {eff }} \\
(\mathrm{K})\end{array}$ & $\begin{array}{c}{[\mathrm{Fe} / \mathrm{H}]} \\
(\mathrm{dex})\end{array}$ & $\begin{array}{c}\log g \\
(\mathrm{dex})\end{array}$ & $\begin{array}{c}V \sin i \\
\left(\mathrm{~km} \mathrm{~s}^{-1}\right)\end{array}$ \\
\hline Daubechies $k=4$ & 5983 & 0.11 & 4.50 & 17.54 \\
Daubechies $k=20$ & 5975 & 0.11 & 4.36 & 17.55 \\
Harr $k=2$ & 5962 & 0.11 & 4.34 & 17.52 \\
bspline $k=20$ & 5961 & 0.10 & 4.36 & 17.77 \\
\hline
\end{tabular}

Notes. Each analysis was performed on WASP-7 using the same method used in Sect. 5.
Table 5. Regional performance of the wavelet method on WASP-20 using a variety of wavelength ranges.

\begin{tabular}{lcccc}
\hline \hline Range & $\begin{array}{c}T_{\text {eff }} \\
(\mathrm{K})\end{array}$ & $\begin{array}{c}{[\mathrm{Fe} / \mathrm{H}]} \\
(\mathrm{dex})\end{array}$ & $\begin{array}{c}\log g \\
(\mathrm{dex})\end{array}$ & $\begin{array}{c}V \sin i \\
\left(\mathrm{~km} \mathrm{~s}^{-1}\right)\end{array}$ \\
\hline $450-500 \mathrm{~nm}$ & 5984 & -0.17 & 4.31 & 3.98 \\
$500-550 \mathrm{~nm}$ & 6076 & -0.06 & 4.33 & 3.76 \\
$550-600 \mathrm{~nm}$ & 5530 & -0.34 & 4.00 & 3.60 \\
$600-650 \mathrm{~nm}$ & 6099 & -0.12 & 4.96 & 3.45 \\
$400-600 \mathrm{~nm}$ & 5983 & -0.11 & 4.50 & 3.63 \\
D15 & 6030 & 0.13 & 4.23 & 4.30 \\
\hline
\end{tabular}

Notes. No priors for $\log g$ were used. $50 \mathrm{~nm}$ windows had $2^{15}$ samples and the $200 \mathrm{~nm}$ windows had $2^{17}$ samples. All were subject to the same analysis in Sect. 5 with no priors on $\log g$.

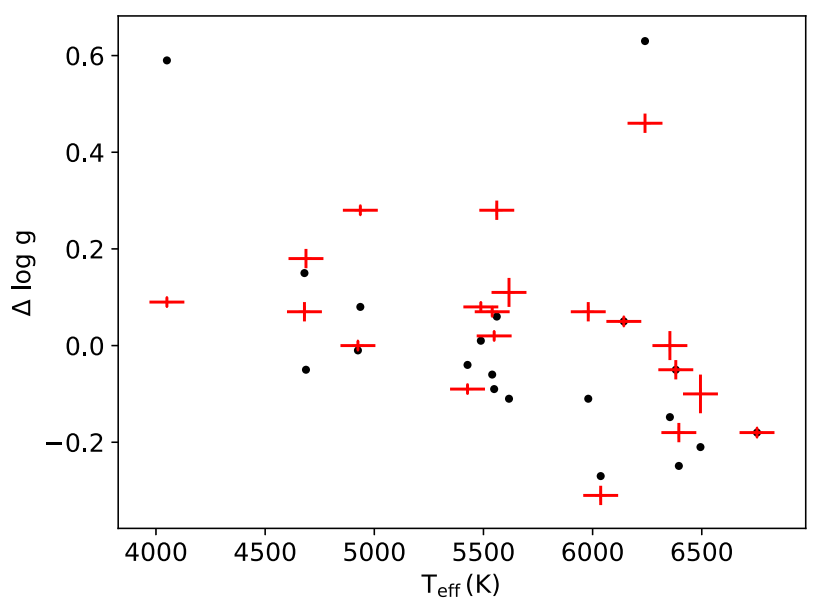

Fig. 9. Difference between spectroscopic $\log g$ and photometric $\log g$ $\left(\log g_{\mathrm{ph}}-\log g_{\mathrm{sp}}\right)$ correlated with $\mathrm{T}_{\mathrm{eff}, \text { wavelet }}$ from this work (black) and from D15 (red).

using the following correction for the $[\mathrm{Fe} / \mathrm{H}]$ values measured with the wavelet method to make them consistent with the metallicity scale of D15:

$[\mathrm{Fe} / \mathrm{H}]_{\text {corrected }}=[\mathrm{Fe} / \mathrm{H}]_{\text {measured }}+0.18$.

\subsubsection{Systematic trend in $\log g$}

We also observe a negative correlation between residual $\log g$ measurements (wavelet - D15) and $\log g$ measured with the wavelet method (Fig. 8c). This trend is observed with and without Gaussian priors on $\log g$ from transit photometry. We calculate a Pearson correlation coefficient of -0.501 for measurements with no $\log g$ prior, suggesting a significant negative correlation. We fit this trend with a first-order polynomial and found a gradient of -0.692 and a y-intercept of 3.067. This correlation evaluates to zero at a wavelet $\log g$ value of 4.44. In principle, the following correction can be used to bring our $\log g$ measurements into line with those from D15,

$\log g_{\text {corrected }}=\log g_{\text {wavelet }}-3.067+0.692 \times \log g_{\text {wavelet }}$.

Without knowing the exact cause of this trend, and given the sensitivity of our $\log g$ estimates to the continuum placement, we are reluctant to advise applying this correction and conclude that the wavelet method cannot reliably estimate $\log g$ 


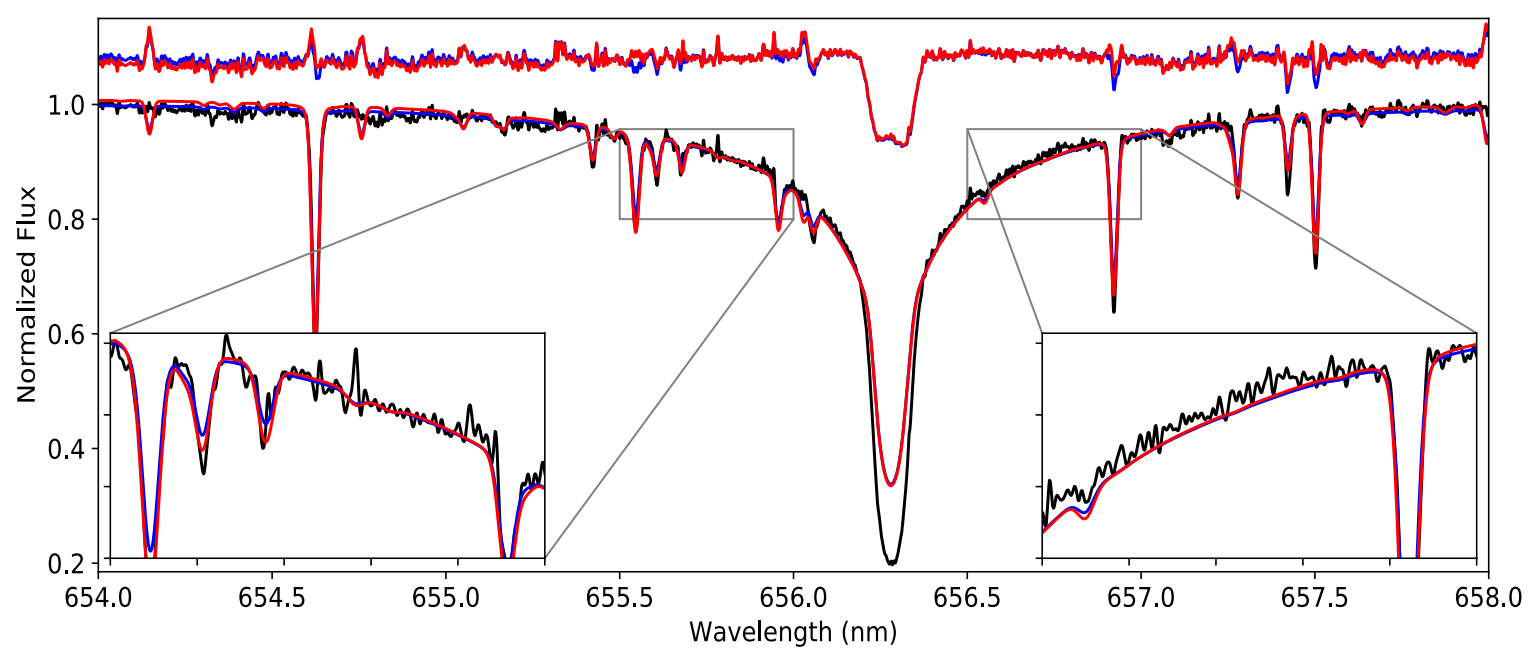

Fig. 10. H- $\alpha$ region for WASP-20 (black) fitted with the best fitted model from D15 (red) and the best model from this work (blue). The near horizontal lines at flux 1.2 are the residuals between the D15 model (red) or wavelet model (blue) and the spectrum of WASP-20.

beyond confirming a dwarf-like surface gravity. Obtaining $\log g$ from a spectrum is typically done through ionization balance (balancing the iron abundance measured from the Fe I and Fe II lines). It is also possible to measure $\log g$ by fitting the wings of gravity sensitive lines (e.g. $\mathrm{Mg}, \mathrm{Na}$ ) using model spectra (the synthesis method). This is essentially how the wavelet method operates (in wavelet space rather than normalised flux space). Accurate determinations of $\log g$ from the synthesis method requires detailed element-abundance measurements for gravity-sensitive $\mathrm{Na}$ and $\mathrm{Mg}$ lines. Estimating the abundances of these elements by scaling from the solar abundance values and applying some correction for $\alpha$ element enhancement will lead to a systematic error in $\log g$ that is difficult to quantify in individual cases. To investigate this further requires another set of comparison stars with independent $\log g$ values (preferably from binary systems where $\log g$ can be accurately measured and not planet transiting systems).

\subsubsection{Precision of atmospheric parameters}

The high precision of the parameters in Table 6 shows that the wavelet method can reliably converge to a well-determined set of atmospheric parameters, but to make use of these parameters we also require a reliable estimate of their true precision that accounts for additional uncertainties due to systematic errors in the data and the models. To obtain a realistic estimate of true precision of the parameters from the wavelet method, $\sigma_{\text {wavelet }}$, we compare the results from our method with the correction to $[\mathrm{Fe} / \mathrm{H}]$ described earlier to those from D15. The standard deviation of the residuals between the measured atmospheric parameters made by D15 and from the wavelet method, $\sigma_{\mathrm{D} 15-\text { wavelet }}$, is a combination of the uncertainties from methods added in quadrature:

$\sigma_{\text {D15-wavelet }}^{2}=\sigma_{\mathrm{D} 15}^{2}+\sigma_{\text {wavelet }}^{2}$,

where $\sigma_{\mathrm{D} 15}$ is the quoted error on the atmospheric parameters from D15. There are two extreme cases: the first is that the uncertainty from D15 is negligible (or at least much better than what we can achieve) giving $\sigma_{\mathrm{D} 15-\text { wavelet }}^{2} \approx \sigma_{\text {wavelet }}^{2}$; and the second is that the inter-method discrepancy, $\sigma_{\mathrm{D} 15-\text { wavelet }}^{2}$, is negligible leaving uncertainties similar to those quoted by D15. In reality, the absolute uncertainty for the wavelet method is somewhere between these two extremes. We adopt a true precision of each parameter from Table 3 using a uniform prior on $\log g$ which is to assume that $\sigma_{\mathrm{D} 15}\left\langle\left\langle\sigma_{\text {wavelet }}\right.\right.$. We suggest applying a correction of +0.18 dex to $[\mathrm{Fe} / \mathrm{H}]$ and not to apply a correction to $\log g$. This means precision of $85 \mathrm{~K}$ for $T_{\mathrm{eff}}, 0.06 \mathrm{dex}$ for $[\mathrm{Fe} / \mathrm{H}]$ and $1.35 \mathrm{kms}^{-1}$ for $V \sin i$. The resulting value of $\log g$ is not likely to be reliable but is good enough to confirm dwarf-like gravity around $\log g=4-5$ dex. We note that these values are comparable to other methods (e.g. Bruntt et al. (2010)).

\subsubsection{Spectrum quality}

In Fig. 11 we plot the difference between atmospheric parameters obtained with the wavelet method (with no priors for $\log g$ ) to those from D15 as a function of $\mathrm{S} / \mathrm{N}$. The sample falls into two categories of quality (those with $S / N \leq 90$ and those with $S / N \geq$ 120). There is noticeably more scatter in the lower-quality group and suggests that the uncertainty of our atmospheric parameters decreases with a better-quality spectrum. The noise profile of a spectrum depends on observing conditions, properties of the star and the instrument used to make the observations. This is why adding Gaussian noise to a synthetic spectrum until the atmospheric parameters are no longer recoverable does not give a true reflection of a methods robustness to noise. Instead, we use 32 (out of 58) observations of the star with the highest $\mathrm{S} / \mathrm{N}$ in the D15 sample - WASP-20. We dyadically split up these spectra and median combine them into different sets. The sets of splits used were 1 spectrum (1 set of 32 spectra), 2 spectra ( 2 sets of 16 spectra), 4 spectra (4 sets of 8 spectra), ..., 32 spectra ( 32 sets each containing just 1 spectra). We scale $\mathrm{S} / \mathrm{N}$ from the coaddition of all 58 spectra:

$S / N=S / N_{58 \text { spectra }} \times \sqrt{\frac{N_{\text {set }}}{58}}$.

Each set was measured with the aforementioned wavelet technique with no prior probability function for $\log g$, and best fitting parameters adopted. The precision and accuracy as a function of $\mathrm{S} / \mathrm{N}$ are shown in Figs. 12 and 13, respectively. We find that systematic errors dominate for a $\mathrm{S} / \mathrm{N}$ below 40. A similar result is found by Smiljanic et al. (2014) who measured UVES-FLAMES spectra for FGK stars from the Gaia-ESO survey and found a systematics threshold of $S / N \approx 50$. 


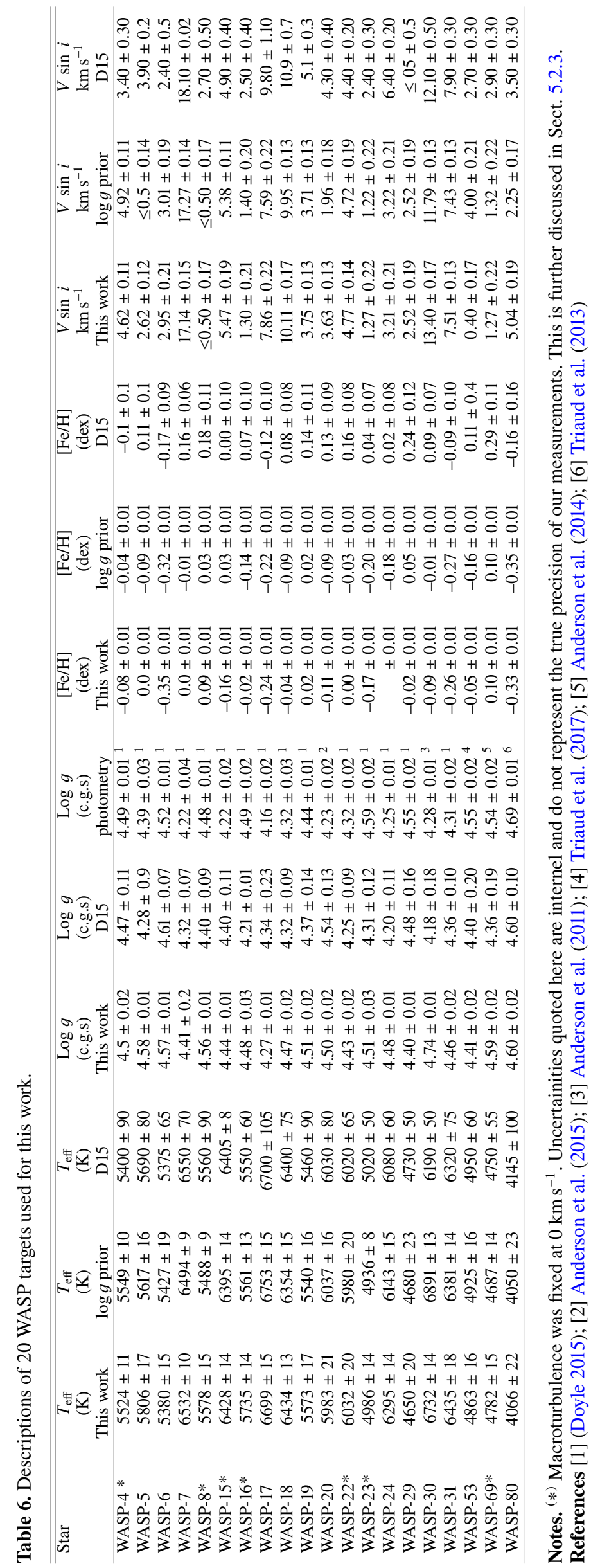




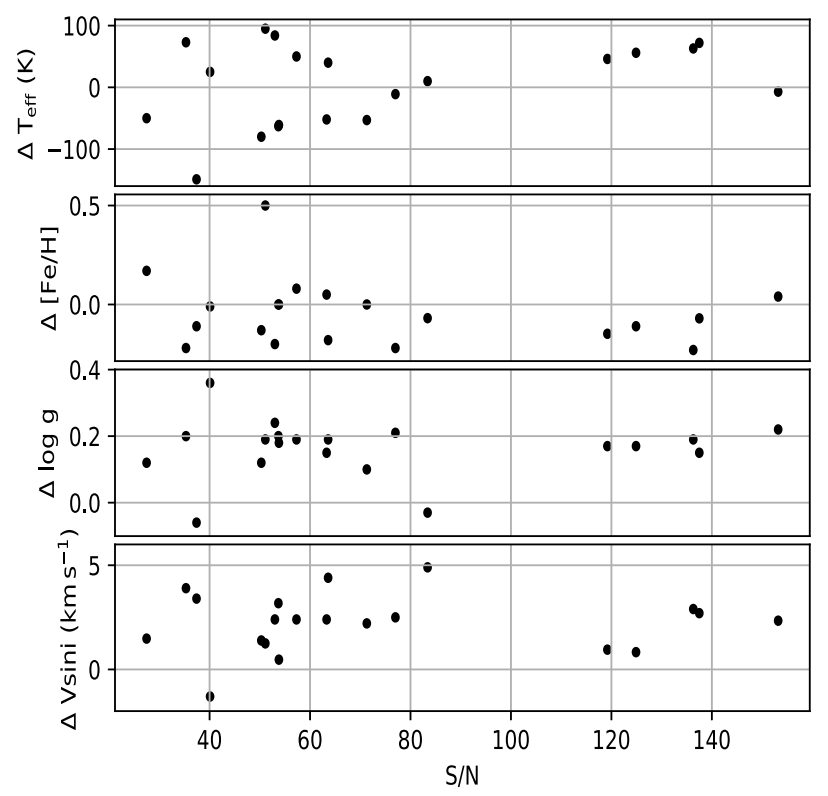

Fig. 11. Atmospheric parameters from the wavelet method, with no prior on $\log g$, compared to those from D15 ( $\left.x_{\mathrm{D} 15}-x_{\text {wavelet }}\right)$ as a function of $\mathrm{S} / \mathrm{N}$.
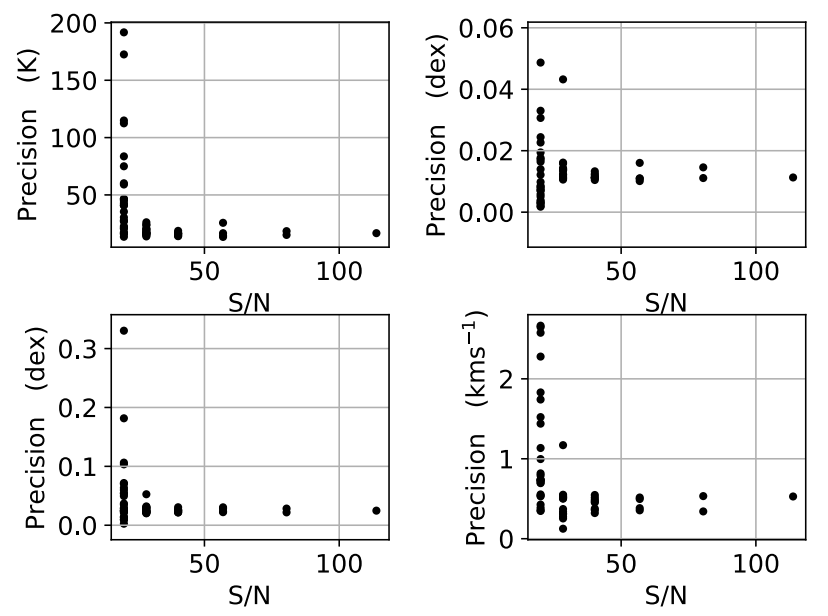

Fig. 12. Precision of the wavelet method versus $\mathrm{S} / \mathrm{N}$ for $T_{\text {eff }}$ (top left), $[\mathrm{Fe} / \mathrm{H}]$ (top right), $\log g$ (bottom left), and $V \sin i$ (bottom right) for WASP-20.

\section{Conclusion}

We have shown that our method accurately recovers the atmospheric parameters of synthetic spectra from a grid of models using subsets of wavelet coefficients in a Bayesian framework. The same method was applied to the CORALIE spectra of 20 FGK stars which have been analysed independently by measurements of EWs from higher-quality HARPS spectra. From this we determine a precision for the parameters derived from the wavelet method of $85 \mathrm{~K}$ for $T_{\text {eff }}, 0.06 \mathrm{dex}$ for $[\mathrm{Fe} / \mathrm{H}]$ and $1.35 \mathrm{kms}^{-1}$ for $V \sin i$. Surface gravity, $\log g$, can also be estimated using our method but it is difficult to assess the precision of this parameter in individual cases. Consequently, we recommend that $\log g$ estimates from our method are only used to decide whether or not a star is a dwarf $(\log g \approx 4.5)$. We find an offset in our metallicity scale compared to the results of Doyle et al. (2013); Doyle (2015) in the sense that our values of $[\mathrm{Fe} / \mathrm{H}]$ are lower by $0.18 \mathrm{dex}$, despite using a consistent
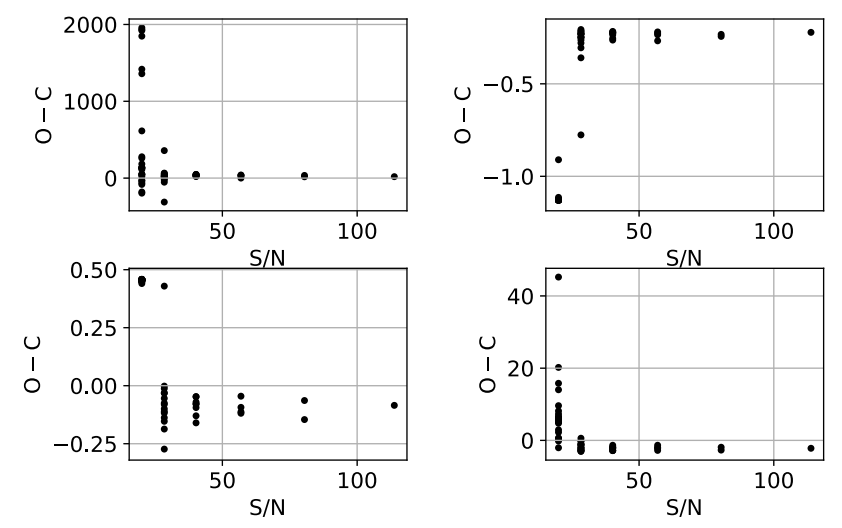

Fig. 13. Accuracy of the wavelet method versus $\mathrm{S} / \mathrm{N}$ for $T_{\text {eff }}$ (top left), [Fe/H] (top right), $\log g$ (bottom left), and $V \sin i$ (bottom right) for WASP-20 using the results from D15 as a zero-point.

solar abundance, and recommend that this offset be applied as a correction to the $[\mathrm{Fe} / \mathrm{H}]$ values from our method. We find our method is robust for échelle spectra with a $\mathrm{S} / \mathrm{N}$ above 40 . Below this value the uncertainity in the measured atmospheric parameters increases to unusable levels. A further development of this method would include a more sophisticated weighting system for the wavelet coefficients beyond the Monte Carlo approach used here.

Our method has already been used to determine the atmospheric parameters of the EBLM J0555-57 (von Boetticher et al. 2017), which hosts one of the densest main sequence stars currently known. This method is also being used to study other EBLM systems and as part of the on-going exoplanet discovery process of the WASP survey. For both exoplanet systems and EBLM binaries, the contribution of the companion star to the optical flux is negligible (they are SB1 binaries) and so our method using models of single stars is appropriate, but it would not be suitable for cases where the companion is detectable in the spectrum (SB2 binaries). We have optimised our method for the application to spectra from CORALIE, but the same method should be equally applicable to spectra with moderate $\mathrm{S} / \mathrm{N}$ from other echelle spectrographs. We have implemented the wavelet method in a python module called waveletspec which is available upon request.

Acknowledgements. SG acknowledges financial support from the Science and Technology Facilities Council (STFC). SG thanks A. H. M. J. Triaud and S. Udry for data acquisition and support. We thank the referee for their careful reading of the manuscript and suggestions that have helped to improve this paper.

\section{References}

Anderson, D. R., Collier Cameron, A., Hellier, C., et al. 2011, ApJ, 726, L19 Anderson, D. R., Collier Cameron, A., Delrez, L., et al. 2014, MNRAS, 445, 1114 Anderson, D. R., Collier Cameron, A., Hellier, C., et al. 2015, A\&A, 575, A61

Anglada-Escudé, G., Amado, P. J., Barnes, J., et al. 2016, Nature, 536, 437 Asplund, M., Grevesse, N., Sauval, A. J., \& Scott, P. 2009, ARA\&A, 47, 481 Baraffe, I., Chabrier, G., Allard, F., \& Hauschildt, P. H. 1998, A\&A, 337, 403 Baranne, A., Queloz, D., Mayor, M., et al. 1996, A\&AS, 119, 373

Belmon, L., Benoit-Cattin, H., Baskurt, A., \& Bougeret, J.-L. 2002, A\&A, 386, 1143

Blanco-Cuaresma, S., Soubiran, C., Heiter, U., \& Jofré, P. 2014, A\&A, 569, A111

Blanco-Cuaresma, S., Nordlander, T., Heiter, U., et al. 2016, in 19th Cambridge Workshop on Cool Stars, Stellar Systems, and the Sun (CS19), 22 Bruntt, H., Bedding, T. R., Quirion, P.-O., et al. 2010, MNRAS, 405, 1907 Dafonte, C., Fustes, D., Manteiga, M., et al. 2016, A\&A, 594, A68 Demory, B.-O., Ségransan, D., Forveille, T., et al. 2009, A\&A, 505, 205 Doyle, A. P. 2015, Ph.D. thesis, Keele University, UK 
Doyle, A. P., Smalley, B., Maxted, P. F. L., et al. 2013, MNRAS, 428, 3164 Doyle, A. P., Smalley, B., Faedi, F., Pollacco, D., \& Gómez Maqueo Chew, Y. 2017, MNRAS, 469, 4850

Essaouabi, A., Regragui, F., \& Ibnelhaj, E. 2009, ArXiv e-prints [arXiv: 0911.0399]

Fernandez, J. M., Latham, D. W., Torres, G., et al. 2009, ApJ, 701, 764

Foreman-Mackey, D., Hogg, D. W., Lang, D., \& Goodman, J. 2013, PASP, 125, 306

Gillon, M., Smalley, B., Hebb, L., et al. 2009, A\&A, 496, 259

Gillon, M., Triaud, A. H. M. J., Demory, B.-O., et al. 2017, Nature, 542, 456

Gómez Maqueo Chew, Y., Morales, J. C., Faedi, F., et al. 2014, A\&A, 572, A50

Goodman, J., \& Weare, J. 2010, Commun. Appl. Math. Comput. Sci., 68, 5

Gray, R. O., \& Corbally, C. J. 1994, AJ, 107, 742

Gustafsson, B., Edvardsson, B., Eriksson, K., et al. 2008, A\&A, 486, 951

Henry, T. J., Jao, W.-C., Subasavage, J. P., et al. 2006, AJ, 132, 2360

Jofré, P., Heiter, U., Worley, C. C., et al. 2017, A\&A, 601, A38

Kervella, P., Mérand, A., Ledoux, C., Demory, B.-O., \& Le Bouquin, J.-B. 2016, A\&A, 593, A127

Kraft, R. P., \& Ivans, I. I. 2004, Origin and Evolution of the Elements (Pasadena: Carnegie Observatories)

Kraus, A., Tucker, R., Thompson, M., Craine, E., \& Hillenbrand, L. 2011, ApJ, 728,48

Li, X., Lu, Y., Comte, G., et al. 2015, ApJS, 218, 3

Manteiga, M., Ordóñez, D., Dafonte, C., \& Arcay, B. 2010, PASP, 122, 608
Morales, J. C., Gallardo, J., Ribas, I., et al. 2010, ApJ, 718, 502

Mortier, A., Santos, N. C., Sousa, S. G., et al. 2013, A\&A, 558, A106

Mortier, A., Sousa, S. G., Adibekyan, V. Z., Brandão, I. M., \& Santos, N. C. 2014, A\&A, 572, A95

Nefs, S. V., Birkby, J. L., Snellen, I. A. G., et al. 2013, MNRAS, 431, 3240

Olkkonen, J. T. 2011, DIscrete Wavelet Transforms, ed. J. T. Olkkonen (InTech) Pollacco, D. L., Skillen, I., Collier Cameron, A., et al. 2006, PASP, 118, 1407

Queloz, D., Mayor, M., Weber, L., et al. 2000, A\&A, 354, 99

Queloz, D., Mayor, M., Udry, S., et al. 2001, The Messenger, 105,

Sedaghati, E., Boffin, H. M. J., Jeřabková, T., et al. 2016, A\&A, 596, A47

Smiljanic, R., Korn, A. J., Bergemann, M., et al. 2014, A\&A, 570, A122

Southworth, J. 2011, MNRAS, 417, 2166

Southworth, J., Mancini, L., Madhusudhan, N., et al. 2017, AJ, 153, 191

Spada, F., Demarque, P., Kim, Y.-C., \& Sills, A. 2013, ApJ, 776, 87

Stumpe, M. C., Smith, J. C., Van Cleve, J., et al. 2012, in AAS Meeting Abstracts, 220, 330.04

Torres, G. 2013, Astron. Nachr., 334, 4

Torres, G., Andersen, J., \& Giménez, A. 2010, A\&ARv, 18, 67

Triaud, A. H. M. J., Queloz, D., Hellier, C., et al. 2011, A\&A, 531, A24

Triaud, A. H. M. J., Anderson, D. R., Collier Cameron, A., et al. 2013, A\&A, $551, \mathrm{~A} 80$

Triaud, A. H. M. J., Neveu-VanMalle, M., Lendl, M., et al. 2017, MNRAS, 467, 1714

von Boetticher, A., Triaud, A. H. M. J., Queloz, D., et al. 2017, A\&A, 604, L6

Zhou, G., Bayliss, D., Hartman, J. D., et al. 2014, MNRAS, 437, 2831 